\title{
Soliton compression to few-cycle pulses by cascaded quadratic nonlinearities
}

\author{
Bache, Morten; Moses, Jeffrey; Bang, Ole; Wise, Frank
}

Published in:

European Conference on Lasers and Electro-Optics, 2007 and the International Quantum Electronics Conference. CLEOE-IQEC 2007.

Link to article, DOI:

10.1109/CLEOE-IQEC.2007.4386319

Publication date:

2007

Document Version

Publisher's PDF, also known as Version of record

Link back to DTU Orbit

Citation (APA):

Bache, M., Moses, J., Bang, O., \& Wise, F. (2007). Soliton compression to few-cycle pulses by cascaded quadratic nonlinearities. In European Conference on Lasers and Electro-Optics, 2007 and the International Quantum Electronics Conference. CLEOE-IQEC 2007. (pp. CF6-4-THU). IEEE. https://doi.org/10.1109/CLEOEIQEC.2007.4386319

\section{General rights}

Copyright and moral rights for the publications made accessible in the public portal are retained by the authors and/or other copyright owners and it is a condition of accessing publications that users recognise and abide by the legal requirements associated with these rights.

- Users may download and print one copy of any publication from the public portal for the purpose of private study or research.

- You may not further distribute the material or use it for any profit-making activity or commercial gain

- You may freely distribute the URL identifying the publication in the public portal 


\title{
Soliton Compression to Few-cycle Pulses by Cascaded Quadratic Nonlinearities
}

\author{
Morten Bache ${ }^{1}$, Jeffrey Moses ${ }^{2}$, Ole Bang ${ }^{1}$, Frank W. Wise ${ }^{2}$ \\ 1. COM•DTU, Technical University of Denmark, Bld. 345v, DK-2800 Lyngby, Denmark. \\ 2. Department of Applied and Engineering Physics, Cornell University, Ithaca, New York 14853.
}

For second-harmonic generation (SHG) the phase mismatch is given by $\Delta k=k_{2}-2 k_{1}$. For $\Delta k>>0$ a large negative Kerr-like nonlinear phase shift may be induced on the fundamental wave (FW). This self-defocusing nonlinearity together with normal dispersion may compress a pulse, and problems normally encountered due to self-focusing in cubic media are avoided. Thus, having no power limit, in bulk media a self-defocusing soliton compressor can create high-energy near single-cycle fs pulses [1]. Here we present a theoretical and numerical investigation of pulsecompression in a nonlinear crystal. We introduce an SHG soliton number and show that compression only takes place when it is larger than the "usual" Kerr soliton number. This can be achieved by adjusting $\Delta k$, but only if the quadratic material nonlinearity is sufficiently stronger than the cubic Kerr nonlinearity. Also the group-velocity mismatch (GVM) between the FW and second harmonic (SH), given by the inverse group velocity difference $d_{12}=1 / v_{g, 1}-1 / v_{g, 2}$, limits the compression regime [1a,1c]. We show that in a typical nonlinear crystal, efficient, good-quality compression down to $<2$ optical cycles may be observed when optimizing $\Delta k$ within the compression regime.

The system is modeled by coupled nonlinear Schrödinger equations including quadratic nonlinear terms and selfsteepening [1d]. The rescaled equations have a characteristic Kerr soliton number $N_{\text {Kerr }}$, similar to the fiber optics one, and a new SHG one $N_{\mathrm{SHG}}$. Because the material Kerr nonlinearity is self-focusing it counteracts the cascaded selfdefocusing nonlinearity. Thus, a requirement for $\mathrm{FW}$ soliton compression is $N_{\mathrm{SHG}}>N_{\mathrm{Kerr}}$, posing an upper limit on $\Delta k$ as

$$
\bar{N}=\frac{N_{\mathrm{SHG}}}{N_{\text {Kerr }}}>1, \quad \Rightarrow \quad|\Delta k|<\Delta k_{c}=\frac{\omega_{1} d_{\text {eff }}^{2}}{c n_{1} n_{2} n_{\text {Kerr }}} \quad N_{\text {Kerr }}^{2}=L_{\mathrm{D}, 1} \frac{2 \omega_{1} n_{\mathrm{Kerr}} I_{1, \text { in }}}{\varepsilon_{0} c^{2} n_{1}}, \quad N_{\mathrm{SHG}}^{2}=L_{\mathrm{D}, 1} \frac{2 \omega_{1}^{2} d_{\text {eff }}^{2} I_{1, \text { in }}}{\varepsilon_{0} c^{3} n_{1}^{2} n_{2}|\Delta k|}
$$

where $L_{\mathrm{D}, 1}$ is the FW dispersion length, $I_{1 \text {,in }}$ the FW input intensity, $n_{\text {Kerr }}$ the Kerr nonlinear refractive index, $d_{\text {eff }}$ the effective quadratic nonlinearity, $n_{j}$ the refractive indices. Notice that $\bar{N}$ is independent on $I_{1, \text { in. }}$ GVM poses another, lower, compression limit: $\Delta k>\Delta k_{\mathrm{sr}}=4 \pi\left|d_{12}\right| / T_{1 \text {,in }}$, where $T_{1 \text {,in }}$ is the FW input pulse duration. In this stationary regime the nonlinear phase shift builds up before GVM separates the fields [1a]. These limits define a $\Delta k$-compression window, which opens $\left(\Delta k_{c}>\Delta k>\Delta k_{\mathrm{sr}}\right)$ when GVM effects are small and $d_{\mathrm{eff}}^{2} / n_{\mathrm{Kerr}}$ is large. An excellent material for compression is $\beta$-barium-borate (BBO) because it has a high $d_{\text {eff }}^{2} / n_{\text {Kerr }}$-ratio. Performing realistic numerical simulations of a BBO for $\lambda_{1}=1060 \mathrm{~nm}$, Fig. 1(a) shows that the transition to compression happens exactly at $\bar{N}=1$. As $\bar{N}$ is increased by reducing $\Delta k$, the output pulse intensity increases until it peaks at position (2) giving a $\sim 12$ fs pulse (compression factor $f_{\text {comp }} \sim 17$ ) containing $45 \%$ of the input energy, see (2) in Fig. 1(b). For larger $\bar{N}$, the pulse splits up, see (3) in Fig. 1(b). In fact, (2) represents the optimal compression point $z_{\max }$ for this input pulse and crystal length $L=50 \mathrm{~mm}$. $z_{\max }$ depends strongly on the input pulse parameters as shown in Fig. 1(c). A characteristic hyperbolic behavior is observed as $\bar{N}$ is varied, with $z_{\max }$ diverging at the transition point $\bar{N}=1$. The corresponding pulse duration at $z=z_{\max }$ drops as $\bar{N}$ is increased, and is minimum at $\bar{N}=1.65\left(\Delta k=50 \mathrm{~mm}^{-1}\right)$, where as the inset shows a clean 6.6 fs pulse is seen $\left(\sim 1.9\right.$ optical cycles, $f_{\text {comp }} \sim 30$, containing $31 \%$ of the input energy). Beyond this point the pulse duration increases again, firstly because such large $\bar{N}$ values require $\Delta k$ small so $\Delta k \sim \Delta k_{\mathrm{sr}}$, and secondly because GVM induces a Raman-like effect with characteristic time $T_{\mathrm{R}, \mathrm{SHG}}=2\left|d_{12}\right| / \Delta k[1 \mathrm{c}]$ resulting in an asymmetric pulse with a reduced pulse compression quality and factor.
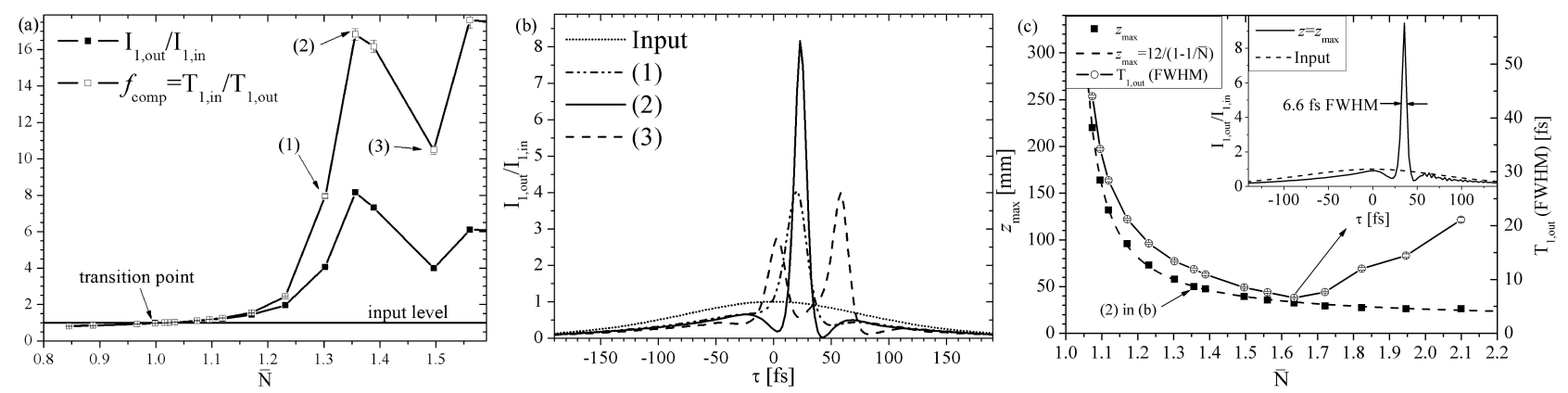

Fig. 1 (a) $I_{1, \text { out }} / I_{1, \text { in }}$ and $T_{1, \text { in }} / T_{1 \text {,out }}$ for a $L=50 \mathrm{~mm}$ BBO crystal. (b) Selected output pulses from (a). (c) $z_{\max }$ and corresponding pulse duration vs. $\bar{N}$. Input pulse: $200 \mathrm{fs}$ FWHM, $I_{1, \mathrm{in}}=50 \mathrm{GW} / \mathrm{cm}^{2} . \Delta k_{\mathrm{sr}}=10 \mathrm{~mm}^{-1}$ and $\Delta k_{c}=137 \mathrm{~mm}^{-1}$.

To conclude, pulse compression with cascaded quadratic nonlinearities requires that the ratio of the SHG and Kerr soliton numbers $\bar{N}>1$. A moderate $\bar{N}$ optimizes the compression. Similar results hold in the fiber case, where we may use the dispersion control offered by photonic-crystal fibers [2] to investigate compression when GVM is reduced.

\section{References}

1. (a) X. Liu, L. Qian, and F. W. Wise, Opt. Lett. 24, 1777 (1999); (b) S. Ashihara et al., J. Opt. Soc. Am. B 19, 2505

(2002); (c) J. Moses and F. W. Wise, Opt. Lett. 31, 1881 (2006); (d) Phys. Rev. Lett. 97, 073903 (2006).

2. M. Bache, H. Nielsen, J. Lægsgaard and O. Bang, Opt. Lett. 31, 1612 (2006). 